\title{
CUBIERTAS AUTOPORTANTES CIRCULARES APLICANDO EL MÉTODO DE ELEMENTOS FINITOS
}

\section{CIRCULAR SELF-SUPPORTING ROOFS APPLYING FINITE ELEMENTS METHOD}

\author{
Cristhian Daniel, Páez Redrován ${ }^{\text {; David Patricio, Guerrero Cuasapaz }}{ }^{2}$ \\ Recibido 05/11/2021: Aprobado: 15/12/2021
}

DOI: https://doi.org/10.51372/gacetatecnica231.6

\begin{abstract}
RESUMEN
Las naves industriales constituyen estructuras destinadas a varios usos en el comercio o desarrollo de una determinada población, en consecuencia, este tipo de estructuras son muy demandadas en el campo de la ingeniería civil, sobre todo en el área de diseño y construcción; por tal motivo en esta investigación se presenta un análisis de cubiertas autoportantes tipo circular, aplicando el método de elementos finitos bajo la ayuda del software de simulaciones ANSYS Workbench 19.0 utilizando elementos finitos tipo área de primer orden. Las deformaciones verticales obtenidas por secciones transversales originales se compararon con las deformaciones de una sección transversal equivalente en inercia y peso. Se analizaron naves industriales con luces de 10, 20, 30, y $40 \mathrm{~m}$. Se consideró una sección de cubierta autoportante tipo CS 1000-610, material ASTM 653 de metal base SS40 y recubrimiento galvanizado Z275 (G90). La estructura de apoyo está conformada por vigas y columnas tipo cercha de estructura metálica conforme a la metodología LRFD y a las normas NEC 2015, AISI S100-07, AISC341-05. Adicionalmente, se analizaron placas de apoyo que adecuadas este tipo de cubiertas. Se observaron concentraciones de esfuerzos en la región de la placa de apoyo, deformaciones por secciones originales mayores a las deformaciones por secciones equivalentes, y variaciones de peso de cubierta y carga viva, teniendo esta última una variación entre 14,75\% a 29,14 $\%$.
\end{abstract}

Palabras clave: Ansys; cubierta autoportante circular; esfuerzo de fluencia; deformaciones verticales; MEF; tensión Von Mises

\footnotetext{
${ }^{1}$ Cristhian Daniel, Páez Redrován, Departamento de Ingeniería Civil, Universidad Politécnica Salesiana, Quito, Ecuador, 13danielpaez13@gmail.com, ORCID: https://orcid.org/0000-0001-5633-6855

${ }^{2}$ David Patricio, Guerrero Cuasapaz, Ingeniero Civil, Magister en estructuras, Docente investigador en la Universidad Politécnica Salesiana, Ecuador, Correo: dguerrero@ups.edu.ec, ORCID: https://orcid.org/0000$\underline{0002-8547-906 X}$
} 


\section{ABSTRACT}

Industrial buildings are structures for various uses in the trade or development of a certain population, consequently, these types of structures are in high demand in the field of civil engineering, especially in the area of design and construction; For this reason, this research presents an analysis of self-supporting circular-type roofs, applying the finite element method under the help of the ANSYS Workbench 19.0 simulation software using first-order area-type finite elements. The vertical deformations obtained by original cross sections were compared to with the deformations of an equivalent cross section in inertia and weight. Industrial buildings with lights of 10,20,30, and $40 \mathrm{~m}$ were analyzed. A self-supporting roof section type CS 1000-610, material ASTM 653 of SS40 base metal and galvanized coating by galvanizing Z275 (G90) was considered. The support structure is made up of metal structure truss-type beams and columns in accordance with the LRFD methodology and the NEC 2015, AISI S100-07, AISC341-05 standards. Additionally, support plates suitable for this type of roof were analyzed. Stress concentrations were observed in the region of the support plate, deformations by original sections greater than the deformations by equivalent sections, and variations in cover weight and live load, the latter having a variation between $14,75 \%$ to $29,14 \%$.

Keywords: Ansys; circular self-supported roof; vertical deformations; FEM; Stress Von Mises; yield stress

\section{INTRODUCCIÓN}

Las cubiertas autoportantes son un sistema que cumple funciones de elemento estructural y cubierta en el mismo elemento, proporcionando resistencia y rigidez a la estructura; esta combinación elimina el uso de correas o vigas secundarias como cubierta. Protege el interior de los agentes externos, tales como la lluvia u otros agentes climáticos; y en lo que respecta a su aspecto estético es muy atractivo en comparación al sistema de cubiertas habitual, ya que al observarla desde el interior se aprecia un sistema muy limpio [1]. Dependiendo del diseño dichas cubierta, los perfiles utilizados se mantienen rectos o se curvan acorde al arco de una circunferencia [2].

Es muy utilizado en varios países, por ejemplo, varias empresas en España tienen más de dos décadas de experiencia en este ámbito [3] [4] [5]. De igual manera, Colombia ya tiene un gran número de empresas que realizan el diseño y construcción de galpones utilizando este sistema [1] [6]. Sin embargo, existen casos en los que los galpones construidos han sufrido colapsos, tal es el caso de la ubicada en la Parroquia de Puellaro, Pichincha, Ecuador, colapsando en dos ocasiones [7]. En consecuencia, se evidencia que se requieren estudios más detallados respecto a cubiertas autoportantes, ya que utilizan secciones transversales cuya geometría es particular dependiendo de máquina encargada de proporcionar su forma.

Para su análisis estructural se planteó utilizar el método de elementos finitos mediante un software de simulaciones, siendo una herramienta de aproximación de soluciones que divide 
el medio continuo de un dominio en elementos de dimensiones finitas con el cual se puede analizar la respuesta de la estructura [8]. Es útil para poder predecir el rendimiento y posibles fallas de un diseño conocido para un uso planificado; y mejorar el rendimiento, seguridad, costo y peso de dicho diseño. De igual manera, se pueden desarrollar conceptos nuevos y obtener información respecto a estos [9]. Un análisis estructural convencional implica en ocasiones realizar varias simplificaciones que podrían causar un sub-dimensionamiento o sobre-dimensionamiento; por tal motivo, el objetivo fundamental que se trazó en la presente investigación fue el realizar el análisis estructural de cubiertas autoportantes, en las que se detalló la forma exacta de la geometría en cuanto a la sección transversal, utilizando la herramienta computacional de simulaciones ANSYS, utilizando elementos finitos y de esta manera verificar los esfuerzos, deformaciones, producidas en este tipo de estructuras.

\section{DESARROLLO}

\subsection{Materiales y Geometría de Cubierta}

Para la cubierta autoportante, se consideró el perfil original en su sección transversal y otro equivalente que represente las propiedades de éste respecto a su peso y su inercia, siendo ambos casos analizados de manera independiente del resto de la estructura. Para los elementos de apoyo de la cubierta autoportante, se consideró una estructura compuesta por perfiles de acero estructural [10]. El tipo de perfil utilizado fue el CS 1000-610, obtenido por la maquinaria con el mismo nombre, proporcionado por la empresa Sanxing Group [2], cuenta con un engargolado vertical como unión entre éstos, ancho útil de $610 \mathrm{~mm}$ y se conforman mediante bobinas de acero de $1000 \mathrm{~mm}$ de ancho (ver Figura 1).

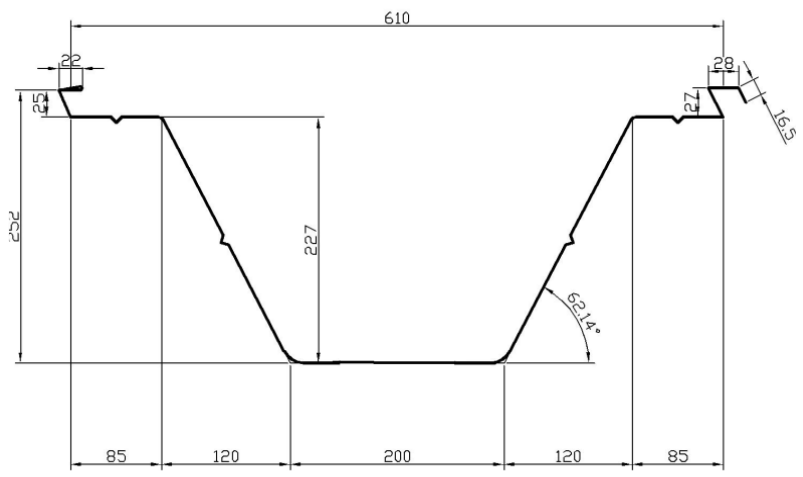

Figura 1. Perfil CS1000-610 utilizado para las cubiertas autoportantes. Fuente: [2]

Estos perfiles utilizan como material bobinas de acero galvanizado ASTM A653 SS40, con su equivalente ecuatoriano NTE INEN 2492 [11]. Se consideró un recubrimiento galvanizado tipo Z275 [12] [13] [14] [15]; y para las vigas y columnas perfiles conformados en frío tipo "C" y "L" de acero A36 con un esfuerzo límite de fluencia de $252 \mathrm{MPa}$. Para todos los 
galpones analizados, se contemplaron naves industriales de tres pórticos sin aleros, con columnas de 6,00 m de altura libre y modulado de pórticos de 6,10 m [16]. Para un número de 21 perfiles se tuvo un ancho de galpón de 12,20 m. Las luces abarcadas (L) variaron de 10, 20, 30 y 40 m como se muestra la Figura 2a y las deformaciones verticales obtenidas fueron comparadas entre cubiertas autoportantes con secciones originales y secciones equivalentes, considerando apoyos articulados de manera independiente del resto de la estructura como se ilustra en Figura $2 b$.

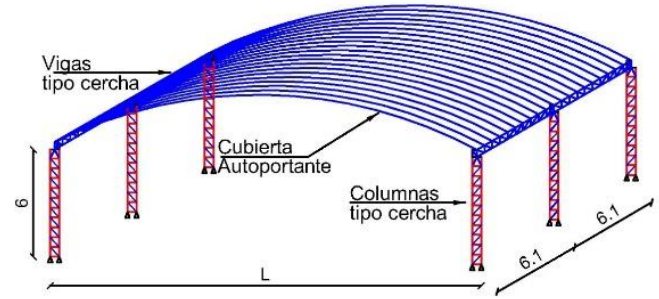

a)

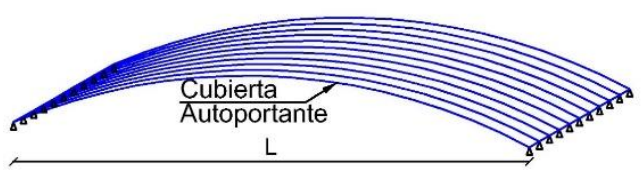

b)

Figura 2. a) Elementos de la cubierta analizada, b) Cubierta autoportante y condición de apoyo para comparación de deformaciones originales y equivalentes. Fuente: los autores

Se analizó la existencia o no, de una variación de peso de carga muerta y carga viva aplicado a la cubierta equivalente y original para un ancho de un vano de la misma. Para la presente investigación, cada vano tuvo $6,10 \mathrm{~m}$, y este espacio fue abarcado por un total de 11 perfiles con un ancho útil de 0,61 m. Adicionalmente, se analizaron las placas de apoyo consideradas para estas cubiertas. Se definió un conjunto de expresiones que representen los parámetros necesarios para su diseño y conformación, como se puede apreciar en la Figura 3.

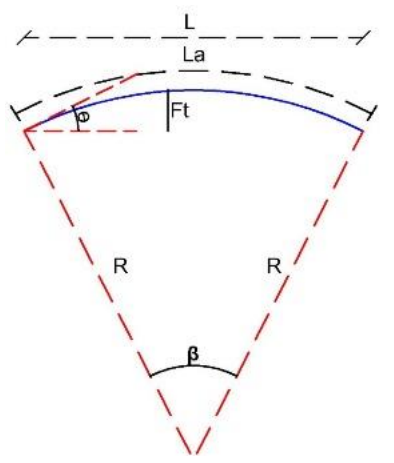

Figura 3. Arco de circunferencia de cubierta autoportante y los parámetros que representan su geometría circular. Fuente: los autores

El radio de la circunferencia de la cubierta $(\mathrm{R})$ se define, como se indica en la ecuación 1.

$$
R=\frac{L^{2}}{8 F t}+\frac{F t}{2}
$$

Donde $(L)$ representa la luz de la cubierta que cubre y $(F t)$ la flecha de la cubierta. La longitud del arco de la cubierta $(L a)$ como se aprecia en la ecuación 2. 


$$
L a=\frac{2 \pi * \operatorname{Ros}^{-1}\left(1-\frac{L^{2}}{28 R^{2}}\right)}{360^{\circ}}
$$

En la ecuación 3 se indica el ángulo comprendido entre el inicio y el fin del $\operatorname{arco}(\beta)$.

$$
\beta=\cos ^{-1}\left(1-\frac{L^{3}}{2 * R^{2}}\right)
$$

Se observa en la ecuación 4, el ángulo de arranque de la cubierta $(\theta)$.

$$
\theta=\frac{\beta}{2}
$$

La selección de la flecha de la cubierta autoportante $(F t)$ se define como se muestra en la ecuación 5.

$$
F t=c * L
$$

Donde $(c)$ es el coeficiente definido como porcentaje que multiplica a la luz de la cubierta a abarcar. Este valor puede variar conforme al diseño arquitectónico hasta un valor de $50 \%$. El valor utilizado fue del $20 \%$ de la luz para todos los casos [1].

\section{METODOLOGÍA}

\subsection{Cargas e Hipótesis de Cargas}

Se aplicó el diseño de última resistencia $(L R F D)$. Esta metodología de diseño multiplica cargas individuales por coeficientes de carga en diferentes combinaciones considerando las incertidumbres propias de la naturaleza, construcción y variabilidad de cargas [17]. La resistencia requerida por el Método LRFD $(R u)$ se define como se muestra la ecuación 6.

$$
R u \leq \emptyset R n
$$

Donde $(R n)$ es la resistencia nominal dada por el material y $(\varnothing)$ es un factor de reducción de resistencia. Se aplicaron siete combinaciones de diseño por resistencia última y una combinación por servicio, como se muestran en las ecuaciones de 7 a 14 [18].

$$
\begin{gathered}
U 2=1.2 D+1.6 L+0.5 \operatorname{máx}(L r, S) \\
U 3=1.2 D+1.6 \operatorname{máx}(L r, S)+\operatorname{máx}(L, 0.5 W(+,-)) \\
U 4=1.2 D+W(+,-)+L+0.5 \operatorname{máx}(L r, S) \\
U 5=1.2 D+E(x(+,-), y(+,-))+L+0.2 S \\
U 6=0.9 D+W(+,-) \\
U 7=0.9 D+E(x(+,-), y(+,-)) \\
\operatorname{Serv}=D+L
\end{gathered}
$$


Donde $(D)$ representa carga muerta, $(L)$ representa carga viva, $(L r)$ representa carga viva de cubierta, $(S)$ representa carga de granizo, $(W)$ representa carga de viento, $(E)$ representa carga por sismo.

\subsection{Sección Equivalente de Cubierta Autoportante}

Se definió un espesor equivalente en inercia para un perfil rectangular y el volumen de esta misma [19]. En la ecuación 15 se indica el espesor equivalente del perfil rectangular plano $\left(e_{e q}\right)$.

$$
e_{e q}=\sqrt[s]{\frac{12 * I_{x-x}}{a}}
$$

Donde $\left(I_{x-x}\right)$ representa la inercia de un perfil de cubierta autoportante, $(a)$ representa el ancho útil del perfil de cubierta autoportante. El volumen del perfil equivalente para un ancho útil y un metro de longitud ( $\left.V o_{l e q}\right)$ se muestra en la ecuación 16.

$$
\mathrm{Vol}_{e q}=e_{e q} * a
$$

Se presenta en la ecuación 17, el peso específico equivalente, considerando el peso del perfil original y el volumen equivalente.

$$
\gamma_{e q}=\frac{W_{\text {cub original }}}{\text { Vol }_{e q}}
$$

Donde ( $\left.W_{\text {cub original }}\right)$ representa el peso real de perfil de cubierta autoportante para un metro de longitud.

\subsection{Apoyo de Perfil de Cubierta Autoportante}

En la Figura 4a, se observan las placas consideradas en las cubiertas de 10 y 20 m de luz, y en la figura $4 \mathrm{~b}$, se pueden apreciar las placas consideradas para los apoyos de 30 y $40 \mathrm{~m}$ de luz. Se utilizaron pernos ASTM A325 y se determinó el número requerido con base en la resistencia al aplastamiento para un perno y la resistencia al corte de una fila de ellos, considerando una junta traslapada y agujeros estándar [19].



a)

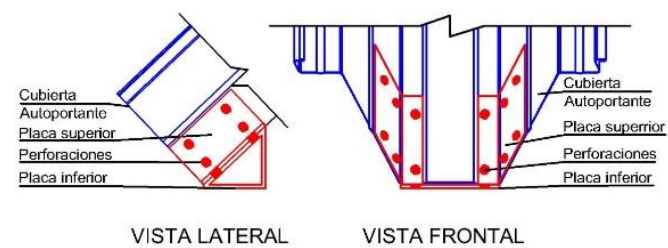

b)

Figura 4. Apoyo del perfil de cubierta autoportante para cubiertas de: a) 10 y $20 \mathrm{~m}$, b) 30 y $40 \mathrm{~m}$. Fuente: los autores

\subsection{Simulación de Cubierta Autoportante en ANSYS Workbench 19.0}

En esta investigación se utilizó el software de simulaciones ANSYS Workbench 19.0 [20]; seleccionándose un análisis estructural estático; mientras que para modelaciones de las cubiertas autoportantes se utilizó el material ASTM 653. Todas las cubiertas analizadas se 
definieron con la geometría del perfil CS 1000-610 indicadas anteriormente en la Figura 1, mediante elementos tipo superficie. No se consideró la aplicación de traslúcidos intermedios en ningún caso. La cubierta de $10 \mathrm{~m}$ tuvo 11 perfiles procesados, como se puede apreciar en la Figura 5a, para la cubierta de $20 \mathrm{~m}$ se procesaron 9 perfiles, como se observa en la figura $5 \mathrm{~b}$. Para la de $30 \mathrm{~m}$ se procesaron 7 perfiles, tal como se indica la figura $6 \mathrm{a}$, y para la cubierta de $40 \mathrm{~m}$ se procesaron 7 como se presenta en la figura $6 \mathrm{~b}$.

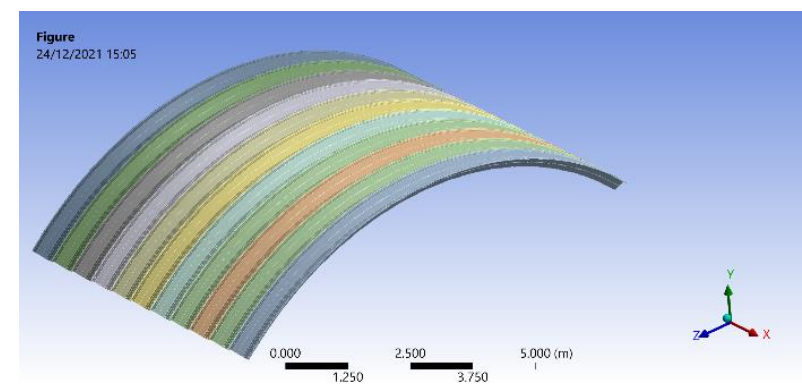

a)

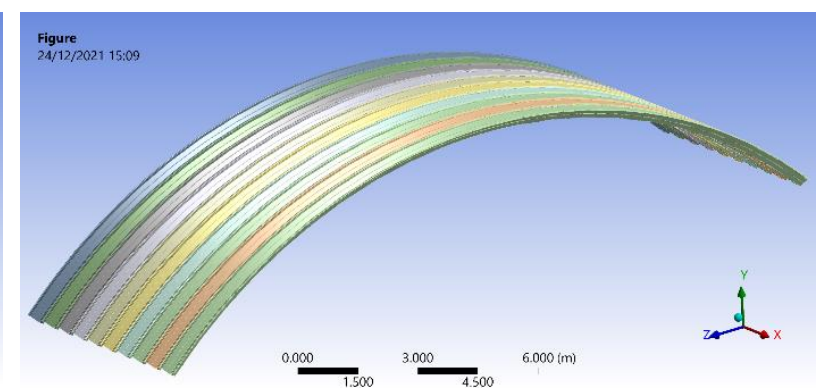

b)

Figura 5. Perfiles procesados en la cubierta de: a) $10 \mathrm{~m}$, b) $20 \mathrm{~m}$. Fuente: los autores

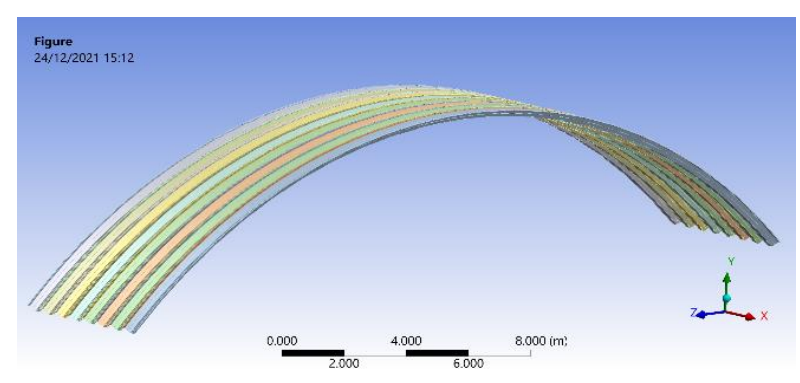

a)

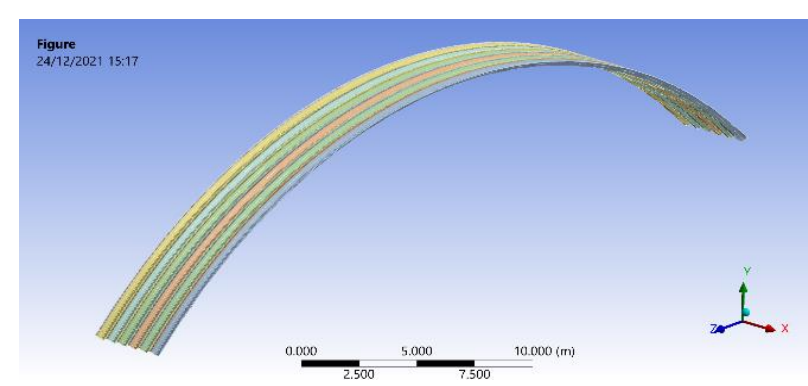

b)

Figura 6. Perfiles procesados en la cubierta de: a) $30 \mathrm{~m}$, b) $40 \mathrm{~m}$. Fuente: los autores

Para la presente investigación se consideró un contacto sin separación en la unión del engargolado, éste consideró un comportamiento lineal respecto a la tipología. Se utilizaron elementos finitos tipo superficie ubicados en el espacio, la forma de dichos elementos se conformó entre cuadriláteros y triángulos con funciones de forma de orden inferior o primarios.

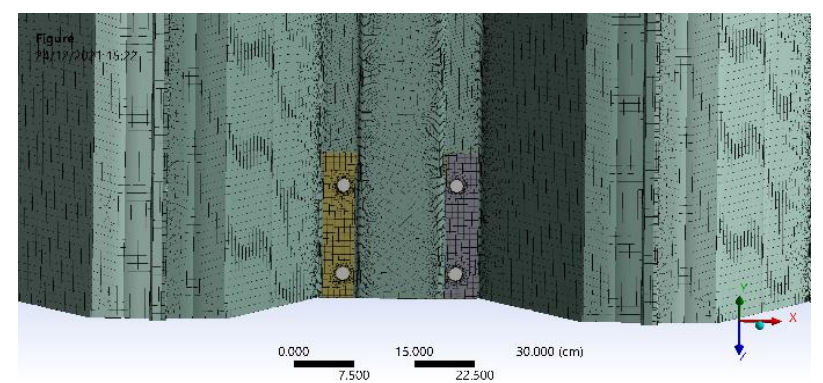

a)

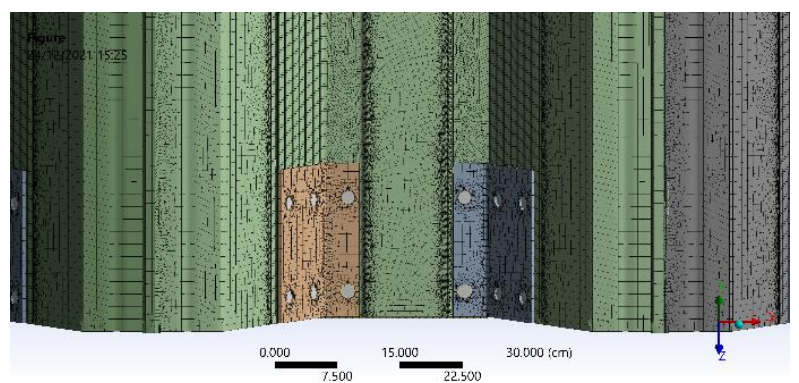

b)

Figura 7. Mallado de perfil de cubierta autoportante para cubiertas de: a) 10 y $20 \mathrm{~m}$, b) 30 y $40 \mathrm{~m}$. Fuente: los autores

En todas las cubiertas autoportantes analizadas se aplicaron diferentes herramientas de control de mallado en las regiones de los resaltes, placas de apoyo, y secciones próximas a los 
engargolados. En la figura 7a, se puede apreciar el mallado aplicado a las cubiertas de 10 y 20 m, mientras que en la figura $7 \mathrm{~b}$, se puede observar el tipo de mallado que se aplicó a las cubiertas de 30 y $40 \mathrm{~m}$. Se consideraron los perfiles de cubierta de manera independiente de las vigas y placas de apoyo. Para la circunferencia formada por la perforación de los pernos se le aplicó una condición de borde sin desplazamientos, además, considerando la interacción de las placas de sujeción y apoyo con el perfil de la cubierta, se aplicó una restricción de desplazamiento vertical en las caras que están en contacto con la placa de apoyo. Para las cubiertas de 10 y $20 \mathrm{~m}$ se consideró el apoyo de la placa inferior como se indica en la figura 4a, representado mediante las restricciones que se pueden apreciar en la figura 8a, y en las cubiertas de 30 y 40 m se contempló el apoyo de la placa inferior, como lo indicado en la figura $4 \mathrm{~b}$ y representado mediante las restricciones se pueden observar en la figura $8 \mathrm{~b}$.

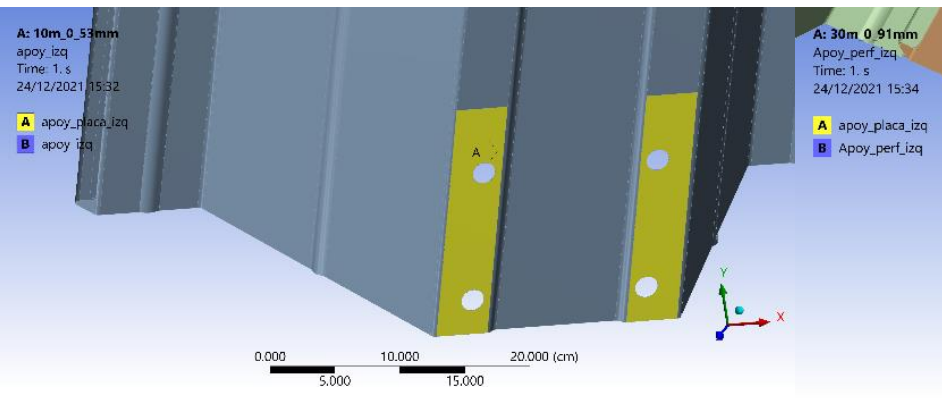

a)

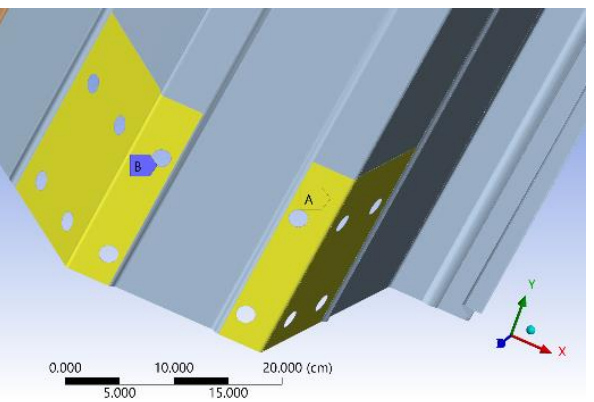

b)

Figura 8. Condición de apoyo en cubiertas de: a) 10 y $20 \mathrm{~m}$, b) 30 y $40 \mathrm{~m}$. Fuente: los autores

\subsection{Simulación de Placa Apoyo de Cubierta Autoportante en ANSYS Workbench 19.0}

Se seleccionó un sistema de análisis estructural estático. Los perfiles de la cubierta autoportante utilizados fueron material ASTM 653, las placas de apoyo se definieron con material ASTM A36 y los pernos utilizados fueron ASTM A325. Además, se consideró la interacción de tres perfiles de cubierta con sus respectivas placas de apoyo. Esto debido a la interacción del engargolado utilizado como traslape entre perfiles de cubierta autoportante. En las figuras 9a y $9 \mathrm{~b}$ se pueden observar los apoyos correspondientes para las cubiertas de $10 \mathrm{y}$ 20 m, así también para luces de 30 y 40 m.

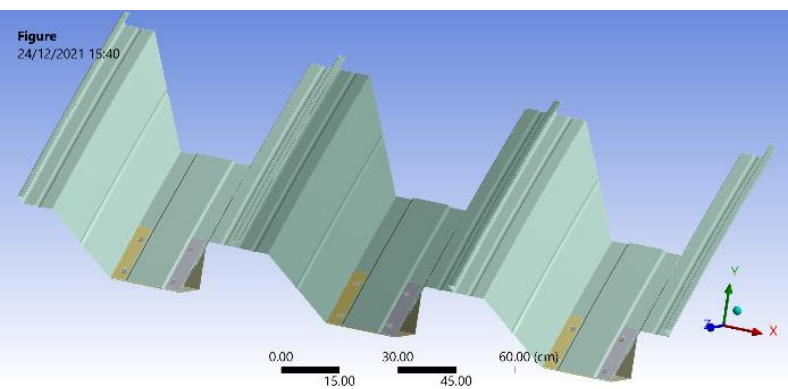

a)



b)

Figura 9. Geometría para la simulación de placas de apoyo en cubiertas de: a) 10 y $20 \mathrm{~m}$, b) 30 y $40 \mathrm{~m}$. Revista Gaceta Técnica. Artículo de Investigación. 23(1), 72-91, enero-julio, 2022 
Para todos los contactos generados entre las diferentes geometrías se consideró un análisis lineal asumiendo un contacto sin separación. Adicionalmente, en todas las perforaciones se aplicaron conexiones tipo barra de sección circular, esta condición representa la interacción de los pernos utilizados. Tanto para los perfiles de cubierta autoportante como para las placas de apoyo, se aplicaron los mismos tipos, funciones de forma y herramientas de control de mallado que los considerados en la simulación de cubiertas independientes de la estructura. En las figuras 10a y 10b se puede apreciar el tipo de mallado que se utilizó para las cubiertas de 10 y 20 m así como también para las cubiertas de 30 y 40 m, respectivamente.

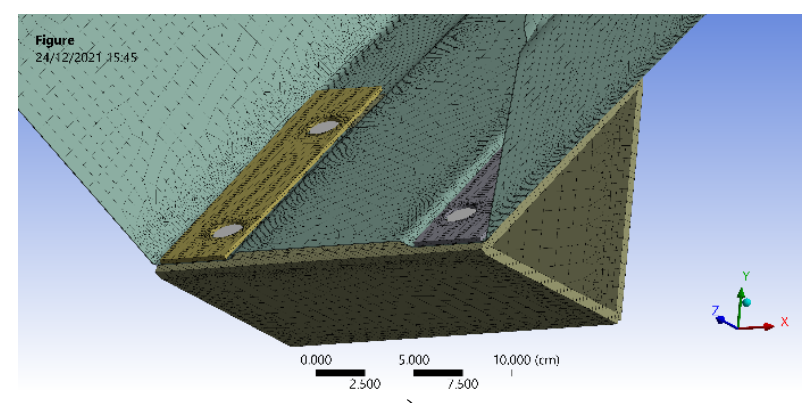

a)

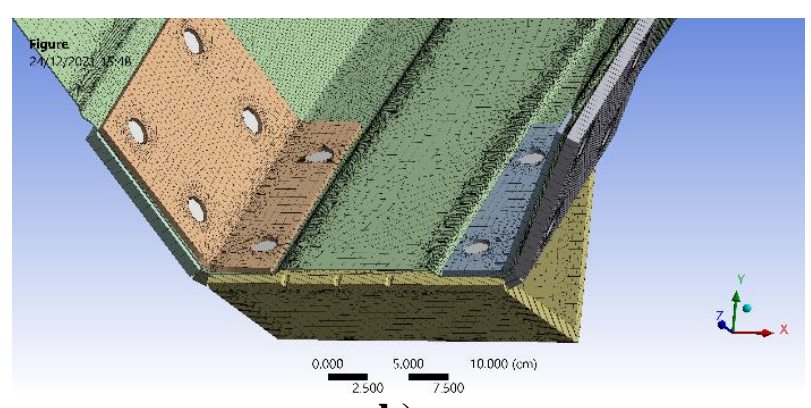

b)

Figura 10. Mallado de placa de apoyo en cubiertas de: a) 10 y $20 \mathrm{~m}$, b) 30 y $40 \mathrm{~m}$. Fuente: los autores Se seleccionaron dos bordes de la placa horizontal de apoyo, en dirección perpendicular de la luz del galpón como se evidencia en la Figura 11. Estos bordes seleccionados no tienen desplazamientos en ninguna dirección. Los pernos fueron modelados como conexiones tipo barra de sección circular con sus diámetros correspondientes según lo indicado en párrafos anteriores y tomando como referencia las Figuras de 9 a 11.

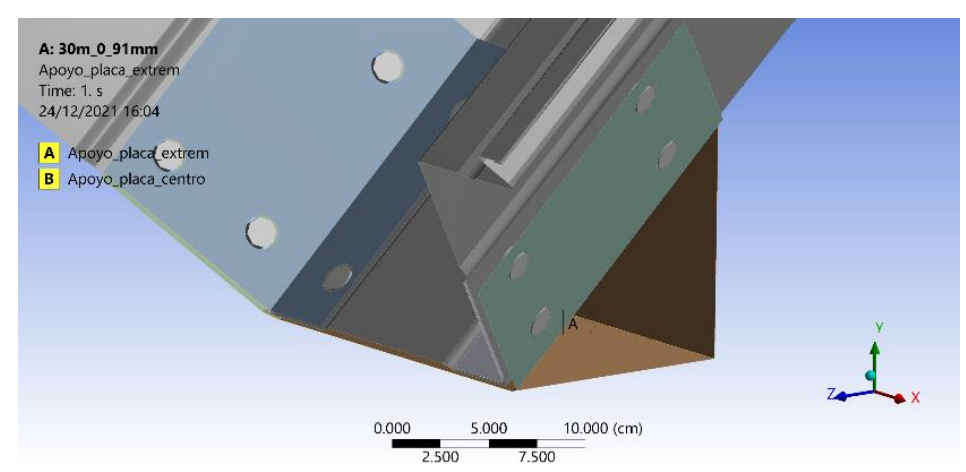

Figura 11. Condición de apoyo de placa seleccionada en el software de simulaciones. Fuente: los autores

\subsection{Tensiones de Von Mises o Esfuerzos Equivalentes}

En el caso de materiales dúctiles, como el acero, se puede utilizar la tensión de Von Mises o esfuerzos equivalentes para poder observar los esfuerzos existentes en un elemento a ser diseñado. Esta metodología considera las tensiones principales que se generan en sus tres ejes ortogonales resultando en una magnitud escalar, como se puede observar en la ecuación 18. 


$$
\sigma_{e}=\sqrt{\frac{1}{2}\left[\left(\sigma_{1}-\sigma_{2}\right)^{2}+\left(\sigma_{2}-\sigma_{3}\right)^{2}+\left(\sigma_{3}-\sigma_{1}\right)^{2}\right]}
$$

Donde:

- $\sigma_{e}$ : esfuerzo equivalente. Considera los esfuerzos principales obtenidos en sus tres ejes ortogonales

- $\sigma 1, \sigma 2, \sigma 3$ : esfuerzos principales obtenidos en sus tres ejes ortogonales

Al utilizar cargas mayoradas por criterio de última resistencia, los esfuerzos equivalentes obtenidos representan los esfuerzos últimos, como se indica en la ecuación 19.

$$
\sigma_{e}=\sigma_{u} \leq \Phi * \sigma_{n}
$$

Donde:

- $\sigma_{u}$ : esfuerzo último requerida por método $L R F D$

- $\Phi$ : coeficiente de reducción de resistencia

- $\sigma_{n}$ : esfuerzo nominal del material

\section{RESULTADOS}

\subsection{Comparación de Deformaciones}

Se analizaron las deformaciones verticales en función de la luz de la cubierta considerada comparando dichas deformaciones entre una sección equivalente con la misma condición de apoyo, y 16 puntos de un perfil de cubierta autoportante original como se puede observar en la Figura 12. Mientras que en la Figura 13 se puede observar la condición de apoyo seleccionada, representa un doble apoyo simple para la comparación de deformaciones verticales entre las cubiertas originales y equivalentes a lo largo de la luz.



Figura 12. Sección original del perfil de cubierta autoportante. Fuente: los autores

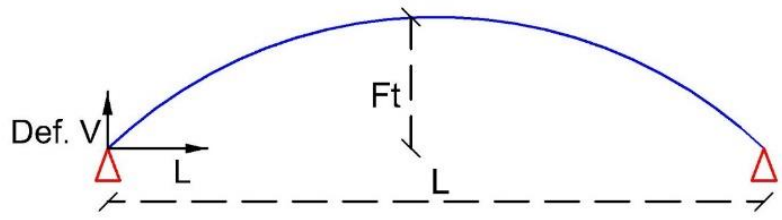

Figura 13. Geometría de cubierta autoportante en la longitud de la luz abarcada y apoyos considerados para análisis de deformaciones verticales. Fuente: los autores

Revista Gaceta Técnica. Artículo de Investigación. 23(1), 72-91, enero-julio, 2022 


\subsection{Comparación de Peso Original y Equivalente de Cubierta para Once Perfiles}

Se obtuvo el peso de carga viva y muerta en la cubierta equivalente para once perfiles. En la ecuación 20 se puede determinar el peso total de cubierta equivalente de once perfiles para una determinada luz de galpón.

$$
D_{\text {Tot eq }}=N * a * L a * e_{e q} * \mathrm{Y}_{e q}
$$

Donde, $(\mathrm{N})$ representa el número de perfiles de cubierta autoportante considerados, para la presente investigación se consideraron once perfiles, $(a)$ es el ancho útil de cada perfil de cubierta autoportante, $(L a)$ es la longitud de arco de cubierta autoportante para una determinada luz de galpón, $\left(e_{e q}\right)$ es el espesor de perfil equivalente de cubierta autoportante, $\left(\gamma_{\text {eq }}\right)$ es el peso específico equivalente de cubierta autoportante. Con la ecuación 21 se puede determinar el peso total de carga viva de cubierta equivalente para once perfiles de cubierta para una determinada luz de galpón.

$$
L r_{\text {Toteq }}=N * a * L a * L r
$$

Donde $(L r)$ representa la carga viva de cubierta. Con la ecuación 22 se pude hallar el peso total de cubierta original para once perfiles de cubierta para una determinada luz de galpón.

$$
D_{\text {Tot Org }}=V_{1 \text { pl orig }} * \mathrm{Y}_{\text {Real }} * N
$$

Donde $\left(V_{1 \text { plorig }}\right)$ es el volumen de un perfil de cubierta original obtenida del software ANSYS, $\left(\mathrm{Y}_{\text {Real }}\right)$ es el peso específico de cubierta que considera su recubrimiento. El peso total de carga viva de cubierta original para once perfiles de cubierta para una determinada luz de galpón ( $L r_{\text {Tot Orig }}$ ) se define con la ecuación 23 .

$$
L r_{\text {Tot Orig }}=A_{1 \text { Pl Orig }} * N * L r
$$

Donde ( $\left.A_{1 \text { Pl Orig }}\right)$ representa el área de aplicación de carga viva de un perfil de cubierta original, obtenido por el software ANSYS. La variación de peso muerto entre carga original y equivalente para un total de 11 perfiles (Var D) y la variación de carga viva entre carga original y equivalente para un total de 11 perfiles (Var $L r$ ), se pueden determinar mediante la aplicación de las ecuaciones 24 y 25.

$$
\begin{gathered}
\text { Var } D=\frac{D_{\text {Tot Orig }}-D_{\text {Tot eq }}}{D_{\text {Tot Orig }}} * 100 \% \\
\text { Var } L r=\frac{L r_{\text {Tot Orig }}-L r_{\text {Tot eq }}}{L r_{\text {Tot Orig }}} * 100 \%
\end{gathered}
$$

\subsection{Verificación de Deformaciones}

Las deformaciones verticales límite fueron consideradas con base al reglamento ASCE/SEI 7- 
16 [21]. En la que se especifica que, para condiciones de servicio se tienen flechas máximas acorde a diferentes condiciones. Para la presente investigación la deflexión máxima permitida se define como lo indicado en la ecuación 26. Donde $(L)$ es la luz que abarca el galpón considerado.

$$
\delta_{V \max p}=L / 200
$$

\subsection{Esfuerzos Obtenidos en Cubierta Autoportante}

En todas las cubiertas autoportantes analizadas en la presente investigación, se encontraron que los esfuerzos máximos se produjeron en las regiones próximas a los apoyos. Al considerar un coeficiente de reducción de resistencia de 0,75 [22] y la resistencia última del material, los esfuerzos producidos en estas regiones no debían superar los $285 \mathrm{MPa}$, como se puede apreciar en las Figuras 14 a 17 y en la Tabla 1.

Tabla 1. Resumen de espesores de perfil y esfuerzos últimos originales máximos de cubierta autoportante. Fuente: los autores

\begin{tabular}{cccc}
\hline Luz $[\mathbf{m}]$ & Espesor $^{\text {a }}[\mathbf{m m}]$ & Esfuerzo máx. $^{\mathbf{b}}[\mathbf{M P a}]$ & Esfuerzo fluencia [MPa] \\
\hline 10 & 0,53 & 131,9 & \\
20 & 0,76 & 272,4 & 275 \\
30 & 0,91 & 215,3 & \\
40 & 1,22 & 162,5 & \\
\hline
\end{tabular}

Espesor final de perfil determinado con base en el diseño

${ }^{\mathrm{b}}$ Esfuerzos máximos obtenidos en la región de la placa

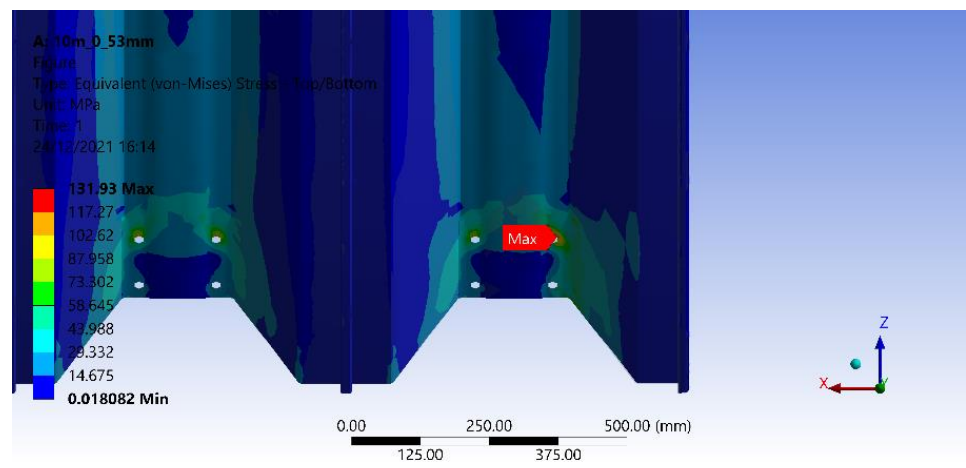

Figura 14. Esfuerzo en zona de apoyo de cubierta de $10 \mathrm{~m}$ con software ANSYS Workbench. Fuente: los autores

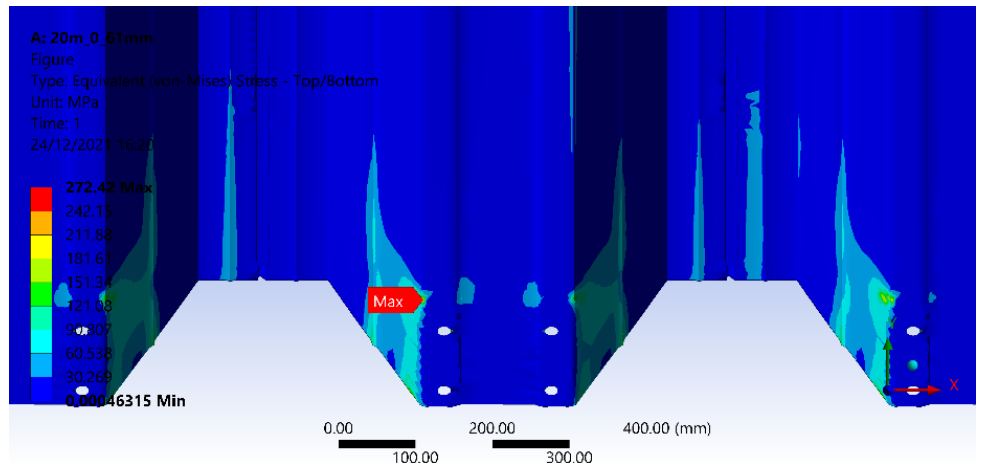

Figura 15. Esfuerzo en zona de apoyo de cubierta de $20 \mathrm{~m}$ con software ANSYS Workbench. Fuente: los autores 


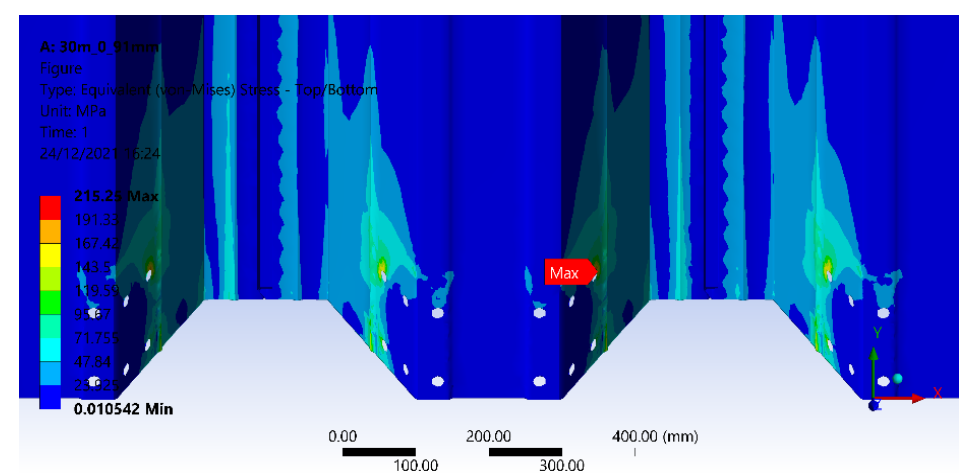

Figura 16. Esfuerzo en zona de apoyo de cubierta de $30 \mathrm{~m}$ con software ANSYS Workbench. Fuente: los autores

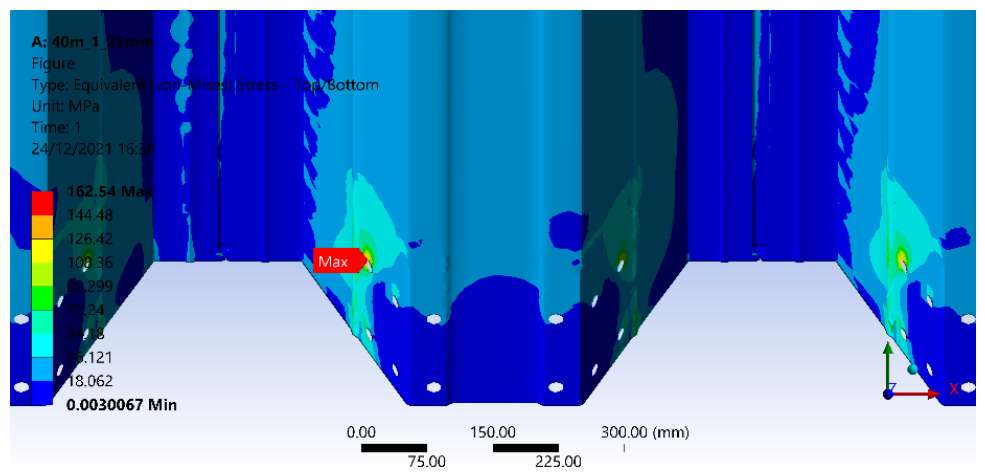

Figura 17. Esfuerzo en zona de apoyo de cubierta de $40 \mathrm{~m}$ con software ANSYS Workbench. Fuente: los autores

\subsection{Esfuerzos Obtenidos en Placas de Apoyo}

Las placas de apoyo están compuestas por acero ASTM A36 con un esfuerzo último de 460 MPa y se consideró un coeficiente de reducción de resistencia de 0.75 [17]. Por tanto, ningún esfuerzo producido en la placa de apoyo debe superar los $345 \mathrm{MPa}$ tal como se indica en la Tabla 2 y se pueden observar las Figuras 18 a 21.

Tabla 2. Resumen de espesores y esfuerzos últimos de placa de apoyo. Fuente: los autores

\begin{tabular}{ccccc}
\hline Luz [m] & Descripción de placa $^{\mathbf{a}}$ & Espesor $^{\mathbf{b}}[\mathbf{m m}]$ & $\begin{array}{c}\text { Esfuerzo } \\
\text { máx. }^{\mathbf{c}}[\mathbf{M P a}]\end{array}$ & $\begin{array}{c}\text { Esfuerzo } \\
\text { máx. fluencia [MPa] }\end{array}$ \\
\hline \multirow{2}{*}{20} & Superior & 2,5 & 224,79 & \\
& Inferior & 5,5 & 310,02 & \\
& Superior & 4,0 & 176,00 & \\
\multirow{2}{*}{30} & Inferior & 8,5 & 318,83 & 460 \\
& Superior & 4,0 & 288,02 & \\
\multirow{2}{*}{40} & Inferior & 8,5 & 256,35 & \\
& Superior & 5,0 & 309,87 & \\
& Inferior & 8,5 & 281,02 & \\
\hline
\end{tabular}

\footnotetext{
${ }^{a}$ Ubicación de la placa sobre o debajo del perfil de cubierta

${ }^{\mathrm{b}}$ Espesor final determinado con base en el diseño

${ }^{\mathrm{c}}$ Esfuerzos máximos obtenidos en la placa de apoyo del perfil
} 


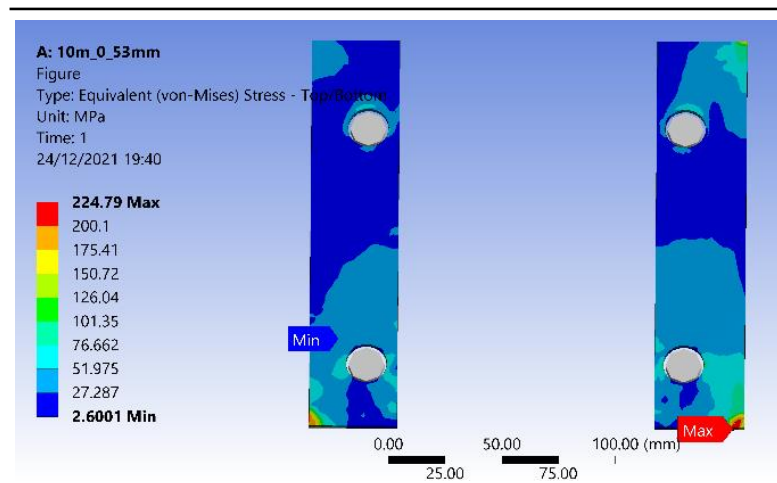

a)

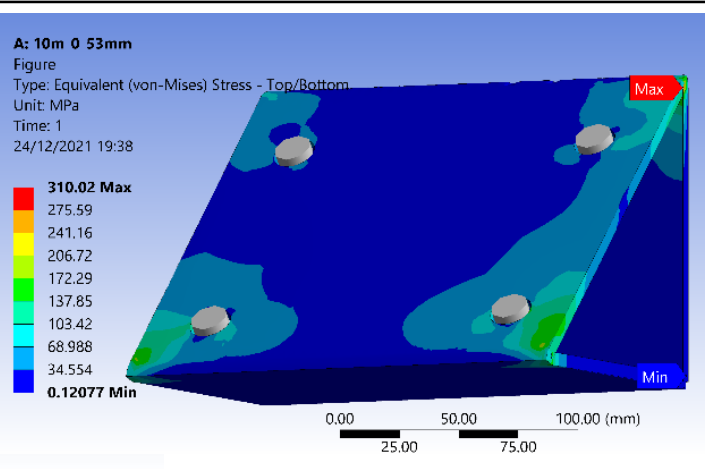

b)

Figura 18. Esfuerzos en la placa de apoyo superior para la cubierta de $10 \mathrm{~m}$. Fuente: los autores

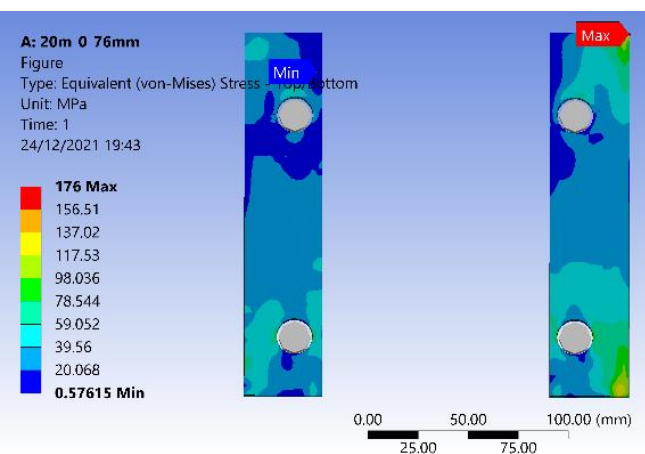

a)

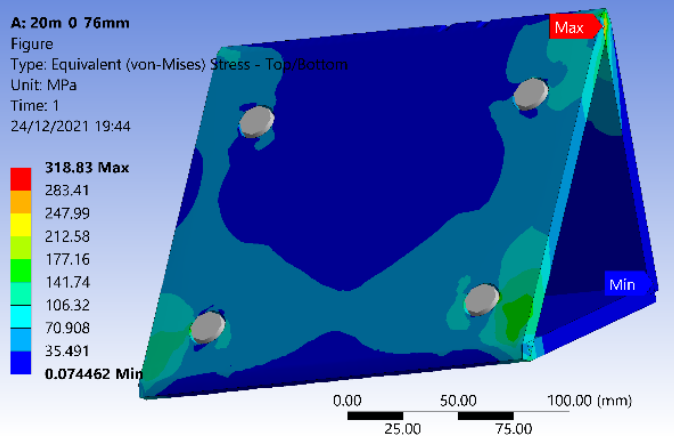

b)

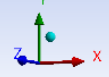

Figura 19. Esfuerzos en la placa de apoyo superior para la cubierta de $20 \mathrm{~m}$. Fuente: los autores

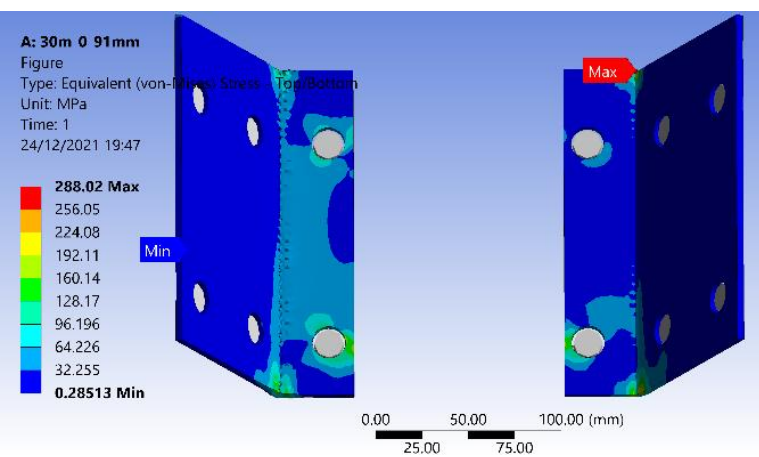

a)



b)

Figura 20. Esfuerzos en la placa de apoyo superior para la cubierta de $30 \mathrm{~m}$. Fuente: los autores

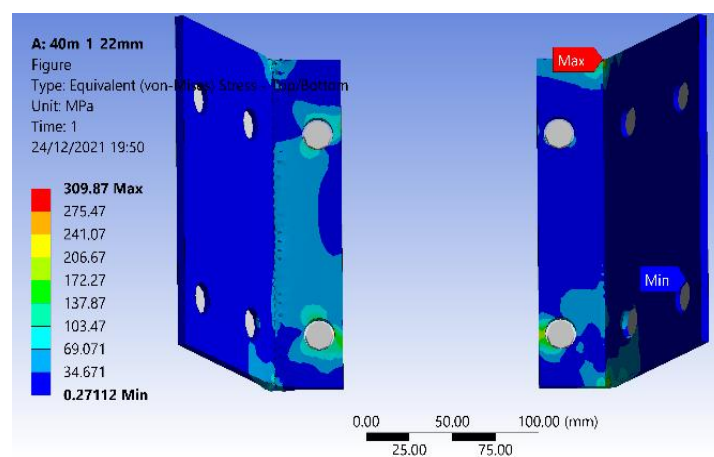

a)

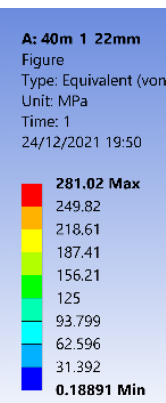

$0.18891 \mathrm{M}$

Figura 21. Esfuerzos en la placa de apoyo superior para la cubierta de $40 \mathrm{~m}$. Fuente: los autores

En la Figura 22 se puede apreciar un esquema de la modelación de la cubierta de $10 \mathrm{~m}$ de luz que se modeló con los pernos. Al considerar pernos tipo ASTM A325 donde las roscas no están excluidas de los planos de corte, se tiene una resistencia nominal a la tensión de 90 
$\mathrm{klb} / \mathrm{pulg}^{2}(620 \mathrm{MPa})$ y una resistencia nominal al cortante en conectores tipo aplastamiento de $54 \mathrm{klb} / \mathrm{pulg}^{2}$ (372 MPa). En consecuencia, lo observó que la resistencia al corte en los pernos cumple con el diseño $L R F D$.

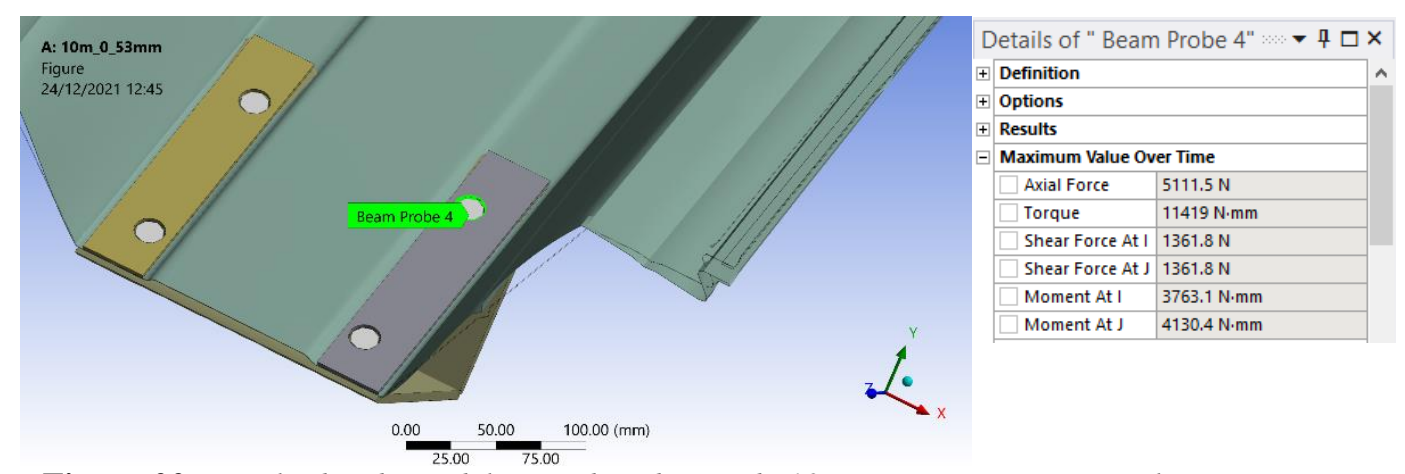

Figura 22. Resultados de modelación de cubierta de $10 \mathrm{~m}$ con pernos. Fuente: los autores

\subsection{Comparación de Deformaciones Verticales}

En todos los casos las máximas deformaciones obtenidas se generaron en los perfiles de los extremos para las cubiertas de sección original y equivalente. Se presentan las gráficas de las comparaciones de deflexiones entre las cubiertas equivalentes y las deflexiones de los puntos identificados en la figura 20 de las cubiertas originales. Los puntos “ $O$ ”, “ $G$ ”, “ $H$ ”, $y$ " $G$ ” de los perfiles extremos tuvieron las deflexiones más grandes para las luces de 10, 20, 30 y $40 \mathrm{~m}$ respectivamente. En las Figuras 23 a 26 se puede apreciar las deformaciones verticales obtenidas para las cubiertas de 10, 20, 30 y $40 \mathrm{~m}$ respectivamente.

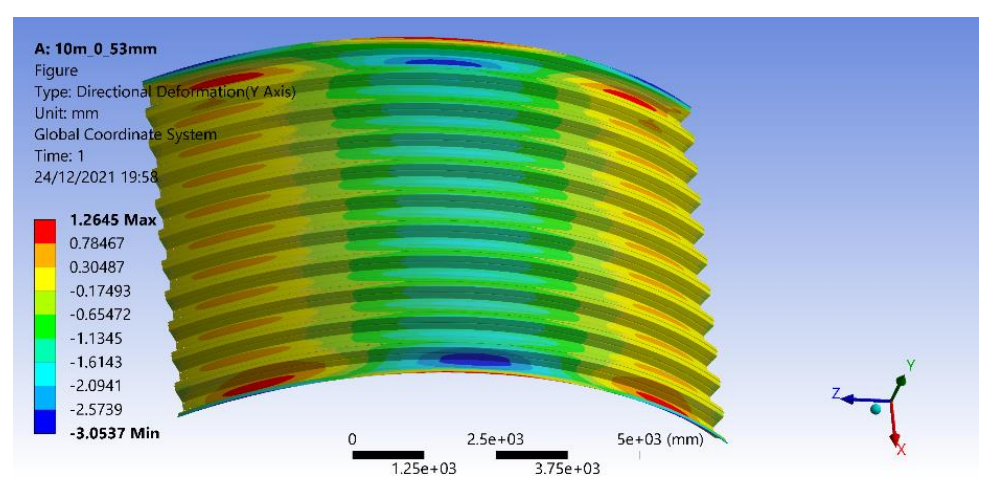

Figura 23. Deformaciones verticales obtenidas de la cubierta original de $10 \mathrm{~m}$ con software ANSYS Workbench. Fuente: los autores

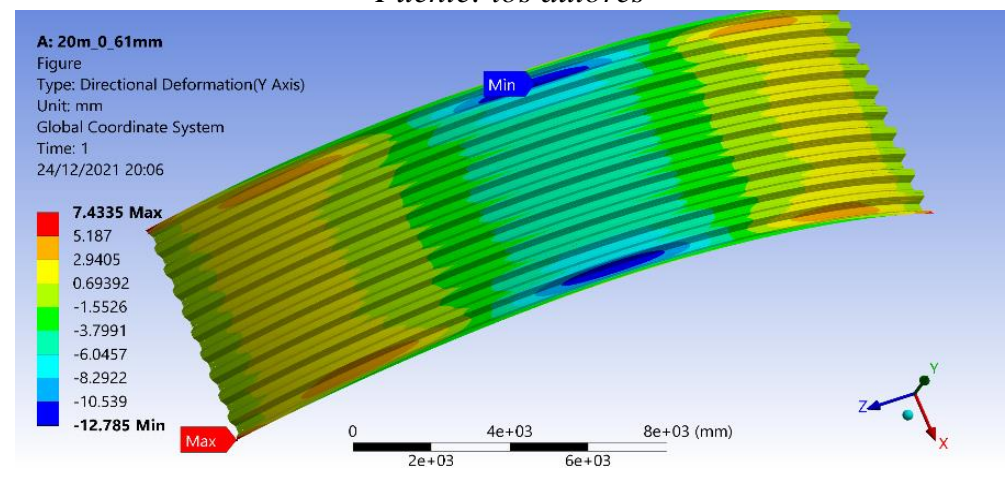

Figura 24. Deformaciones verticales obtenidas de la cubierta original de $20 \mathrm{~m}$ mediante el software ANSYS Workbench. Fuente: los autores 


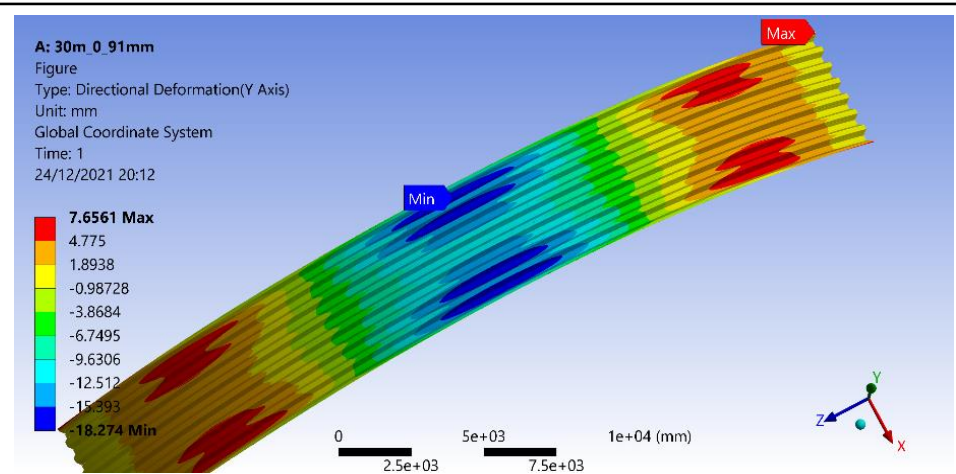

Figura 25. Deformaciones verticales obtenidas de la cubierta original de $30 \mathrm{~m}$ mediante el software ANSYS Workbench. Fuente: los autores

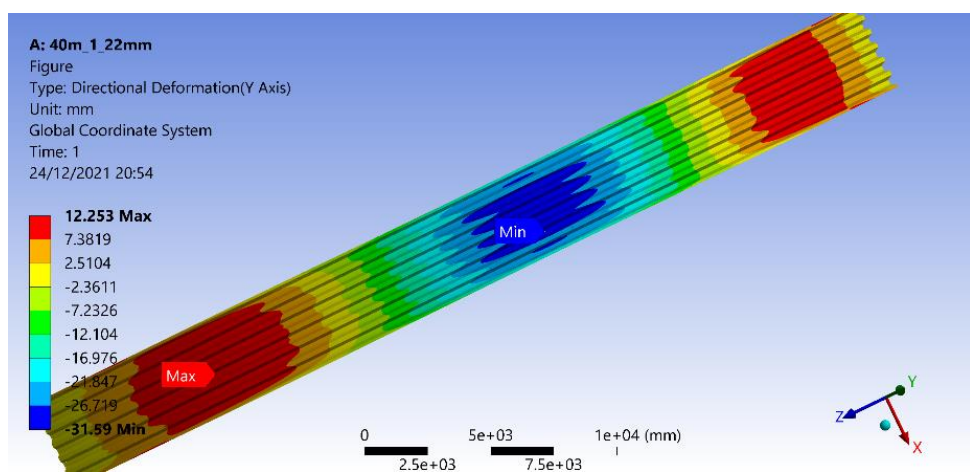

Figura 26. Deformaciones verticales obtenidas de la cubierta original de 40m mediante el software ANSYS Workbench. Fuente: los autores

En las Figuras 27 a 30 se observan las comparaciones de las deformaciones verticales para perfiles originales y equivalentes de las luces indicadas anteriormente.

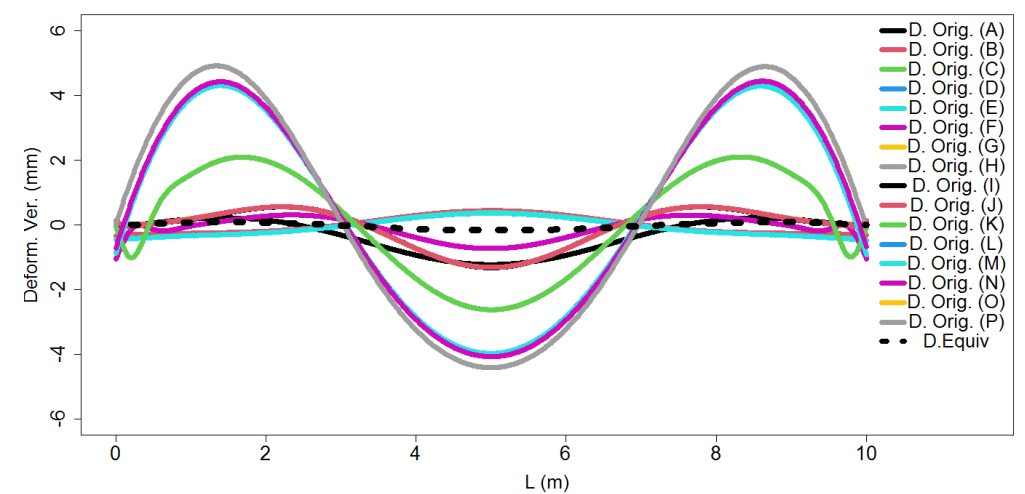

Figura 27. Comparación de deformaciones verticales por perfiles originales y equivalentes para cubierta de 10m. Fuente: los autores

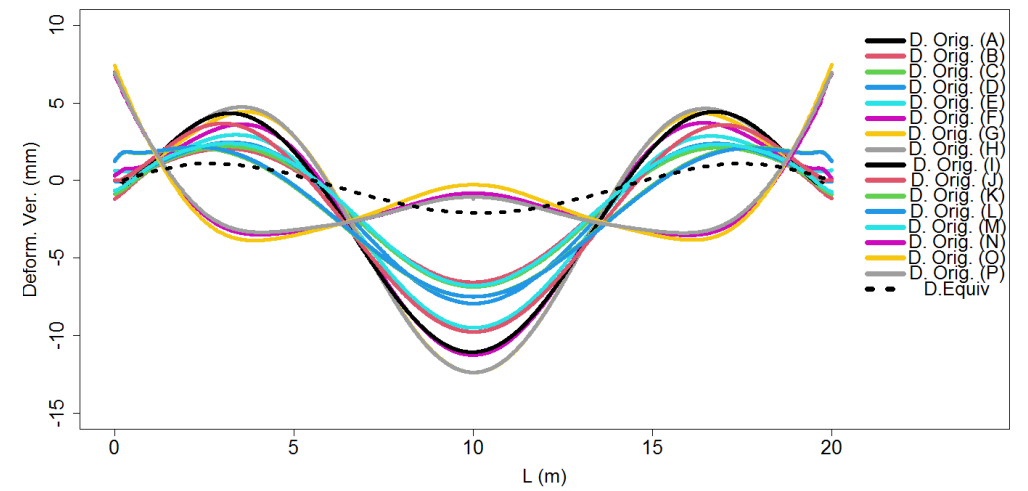

Figura 28. Comparación de deformaciones verticales por perfiles originales y equivalentes para cubierta de $20 \mathrm{~m}$. Fuente: los autores

Revista Gaceta Técnica. Artículo de Investigación. 23(1), 72-91, enero-julio, 2022 


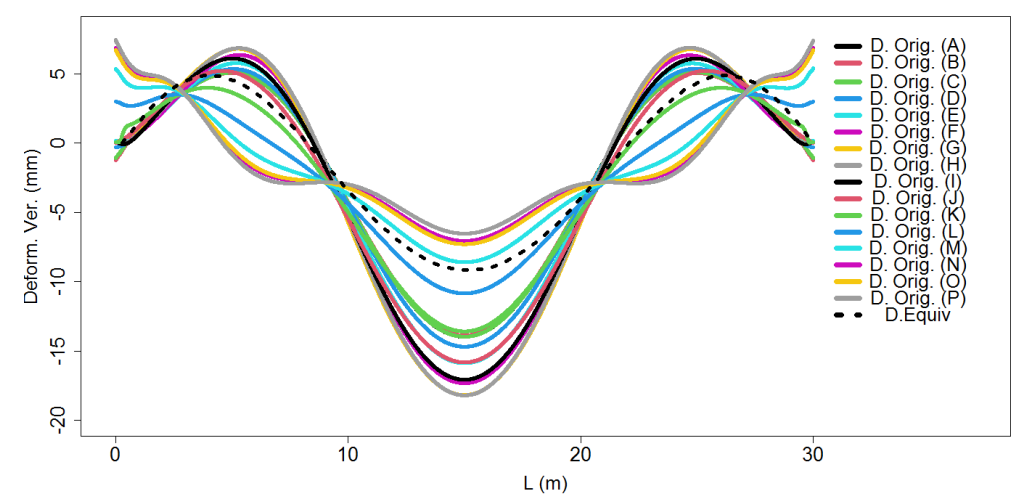

Figura 29. Comparación de deformaciones verticales por perfiles originales y equivalentes para cubierta de 30 m. Fuente: los autores

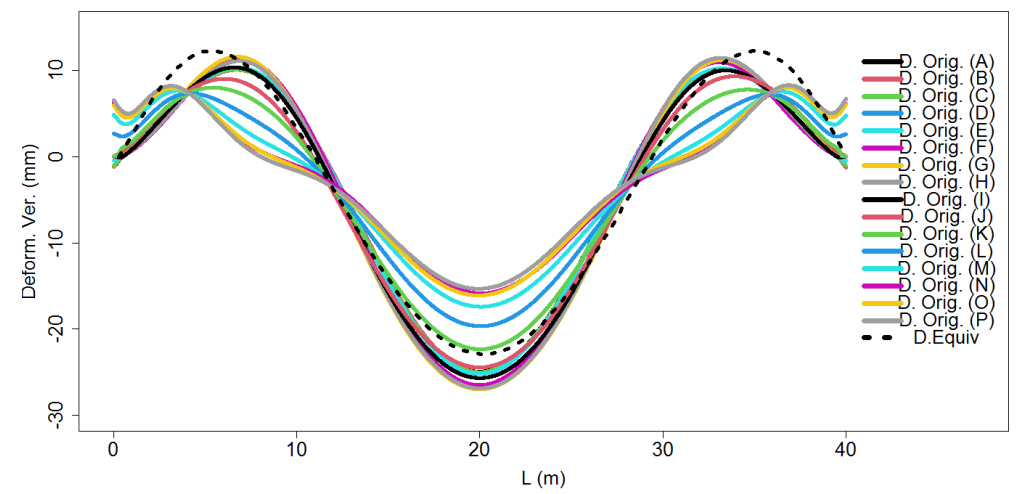

Figura 30. Comparación de deformaciones verticales por perfiles originales y equivalentes para cubierta de 40m. Fuente: los autores

En la Tabla 3 se puede ver la variación entre las deflexiones generadas por cubiertas de perfiles de secciones transversales originales y equivalentes.

Tabla 3. Comparación y variación de deflexiones originales y equivalentes. Fuente: los autores

\begin{tabular}{cccc}
\hline Luz [m] & d. orig a $(\mathbf{m m})$ & d equiv. $^{\mathbf{b}}(\mathbf{m m})$ & Var d orig y d equiv. $^{\mathbf{c}}(\boldsymbol{\%})$ \\
\hline 10 & 3,054 & 0,169 & 94,46 \\
20 & 12,785 & 2,083 & 83,71 \\
30 & 18,274 & 9,155 & 49,90 \\
40 & 31,590 & 22,883 & 27,56 \\
\hline
\end{tabular}

a Deflexiones por cubiertas de sección transversal original

b Deflexiones por cubiertas de sección transversal equivalente

${ }^{\mathrm{c}}$ Variación de deflexiones equivalentes respecto a las originales

\subsection{Comparación de Pesos Totales de Cubierta por Carga Muerta y Viva para Once Perfiles Equivalentes y Originales}

Se obtuvieron las variaciones de pesos totales de carga muerta y viva de cubierta para 11 perfiles equivalentes y originales tal como se puede apreciar en la Tabla 4. 
Tabla 4. Variaciones de pesos por cubiertas originales y equivalentes para once perfiles. Fuente: los autores

\begin{tabular}{ccccccc}
\hline Luz [m] & D eq. $^{\mathbf{a}}(\mathbf{k g})$ & D orig $^{\mathbf{b}}(\mathbf{k g})$ & Lr eq. $^{\mathbf{c}}(\mathbf{k g})$ & Lr orig $\left.^{\mathbf{d}} \mathbf{k g}\right)$ & Var D $^{\mathbf{e}}(\boldsymbol{\%})$ & Var Lr $^{\mathbf{f}}(\boldsymbol{\%})$ \\
\hline 10 & 516 & 521 & 4976 & 5836 & 0,92 & 14,75 \\
20 & 1476 & 1501 & 10107 & 13112 & 1,65 & 22,92 \\
30 & 2646 & 2757 & 15238 & 20198 & 4,05 & 24,56 \\
40 & 4691 & 5007 & 20343 & 28707 & 6,31 & 29,14
\end{tabular}

${ }^{a}$ Peso total de carga muerta de once perfiles de cubierta equivalente

${ }^{\mathrm{b}}$ Peso total de carga muerta de once perfiles de cubierta original

${ }^{c}$ Pero total de carga viva aplicado a once perfiles de cubierta equivalente

d Pero total de carga viva aplicado a once perfiles de cubierta original

e Variación de peso total de carga muerta equivalente respecto a la original

${ }^{\mathrm{f}}$ Variación de peso total de carga viva equivalente respecto a la original

\section{CONCLUSIONES}

El mallado de elementos finitos para las cubiertas autoportantes fue definido con elementos 2D ubicados en el espacio compuesto por triángulos y cuadriláteros, con funciones de forma de orden inferior o primarios. El mallado realizado para las diferentes variantes presentó un formato único para cada luz de galpón. Mientras que, el apoyo para las cubiertas aisladas de la estructura consideró una restricción al desplazamiento horizontal, en el caso de considerar este desplazamiento, se obtendría un desplazamiento excesivo y, por tanto, la falla de la cubierta autoportante.

Al analizar la cubierta completa, se observó que el desplazamiento horizontal fue controlado mediante el uso de tensores, para contrarrestar las deflexiones excesivas. En todos los casos de las cubiertas analizadas, las deflexiones por secciones transversales originales fueron mayores a las deformaciones por secciones transversales equivalentes. Se observaron variaciones de $94,46 \%, 83,71 \%, 49,90 \%, 27,56 \%$ para las cubiertas de 10, 20, 30, y $40 \mathrm{~m}$ respectivamente.

Las placas de apoyo consideradas en las cubiertas de 30 y $40 \mathrm{~m}$ fueron diferentes a las utilizadas en las cubiertas de 10 y $20 \mathrm{~m}$. Esto con el fin de evitar concentraciones de esfuerzos en la zona próxima a la conexión de la hoja del perfil de cubierta autoportante. Existe una considerable variación entre el peso de la carga viva aplicada sobre la sección equivalente y la original, esto se da porque existe más área de aplicación de peso en la cubierta original, estas fueron en el orden de 14,75 \%, 22,92\%,24,56 \%, y 29,14\% para las cubiertas de 10, 20, 30 y $40 \mathrm{~m}$ respectivamente.

Con lo indicado en lo párrafos anteriores, para la presente investigación se puede concluir que al realizar el análisis y diseño de una nave industrial con secciones equivalentes, se obtienen 
deformaciones menores, que no constituyen el comportamiento real que tendrá la estructura, mientras que analizando las cubiertas con su perfil original, se obtienen deformaciones mayores, estas variaciones pueden ocasionar el sub-dimensionamiento de la nave industrial, en el caso de un análisis estructural simplificado y por consiguiente su posible colapso, por lo que es recomendable para este tipo de estructuras tener mayor detalle de su sección transversal para su análisis, diseño y modelado utilizando elementos finitos.

\section{REFERENCIAS}

[1] Proingcol, “Cubiertas autoportantes Colombia Roofing”, Colombia, 2018

[2] Sanxing Group, "Machine manufacturer \& steel building constructor”, China, 1992

[3] Incoperfil, “Cubiertas curvadas autoportantes”, España, 2011

[4] Unitelha, "Ficha técnica perfiles tipo UNTA, Unitelha", España, 1991

[5] Apimet, “Apimet cubiertas autoportantes”, España, 1993

[6] Poliarkit, "Ficha técnica cubierta autoportante sin estructura", Colombia, 2012

[7] M. Guerra, "Comentario desconocimiento del uso de paquetes computacionales, Disponible en: https://www.facebook.com/photo.php?fbid=10215489587937857\&set=pb.13834149 39. $-2207520000 . . \&$ type $=3,2019$

[8] M. Vásquez y E. López, "El método de los elementos finitos aplicado al análisis structural”, España, Editora Noela, 2001

[9] H. Lee, "Finite element simulations with ANSYS workbench 2019", Taiwan, SCD Publications, 2019

[10] E. Arnal, "Proyecto y construcción de galpones modulares", Venezuela, Ed. Sidetur, 2007

[11] NTE INEN 2492, "Láminas de acero recubiertas con zinc (galvanizadas) 0 recubiertas con aleación hierro zinc (galvano-recocido) mediante procesos de inmersión en caliente. Requisitos" Instituto Ecuatoriano de Normalización (INEN), Ecuador, 2009

[12] NTE INEN 2221, "Paneles de acero. Requisitos y métodos de ensayo" Instituto Ecuatoriano de Normalización (INEN), Ecuador, 2016

[13] NTE INEN 1623, "Perfiles abiertos de acero conformados en frío negros o galvanizados para uso estructural. Requisitos e inspección" Instituto Ecuatoriano de Normalización (INEN), Ecuador, 2015

[14] NTE INEN 2526, "Perfiles especiales abiertos, livianos, pregalvanizados y 
conformados en frío para uso en estructuras portantes. Requisitos" Instituto Ecuatoriano de Normalización (INEN), Ecuador, 2010

[15] X. Xhang, "Corrosion and electrochemistry of zinc", New York, Ed. Springer Science+Business Media, 1996

[16] D. Loachamin, A. Freire, D. Guerrero, M. Guerrón, “Análisis técnico-económico de naves industriales mediante interpolación no lineal de Lagrange" Revista técnica de la facultad de ingeniería Universidad del Zulia, 44(2), 104-116, Disponible en: https://doi.org/10.22209/rt.v44n2a05, 2021

[17] F. Crisafulli, "Diseño sismorresistente de construcciones de acero", Chile, Asociación latinoamericana del acero, 2018

[18] NEC-SE-CG, "Norma ecuatoriana de la construcción. Cargas no sísmicas" Norma Ecuatoriana de la Construcción (NEC), Ecuador, 2015

[19] J. McCormac, “Diseño de estructuras de acero”, México, Ed. Alfaomega, 2012

[20] ANSYS Workbench 19.0, "Simulación en ingeniería”, 2018

[21] ASCE 7-16, "Minimum design loads and associated criterio for buildings and other structures” American Society of Civil Engineers (ASCE), E.E.U.U., 2016

[22] AISC 360-16, "Specification for structural Steel buildings" American Institute of Steel Construction (AISC), E.E.U.U., 2016 